\title{
Simulation of the impact response of a sliotar core with linear and non-linear contact models
}

Kevin Manley a, Fiachra Collins b' d, Kevin Cronin a, Edmond Byrne a, Kieran Moran Dermot Brabazon c'd '

\begin{abstract}
Static and dynamic models of the characteristic responses of sliotar cores made of both cork and polyurethane were studied in this work in order to understand their constitutive behaviour. Data from quasi-static tests at $10 \mathrm{~mm} / \mathrm{s}$ and from dynamic impacts at speeds from 5 to $25 \mathrm{~m} / \mathrm{s}$ were used to develop and evaluate the models. The quasi-static response was described well by Hertzian theory. A non-linear HunteCrossley model and a modified linear KelvineVoigt model were used to predict the dynamic response with set mass and shape coefficient parameters. The HunteCrossley model predicted well both the maximum force and maximum deflection for each ball type. The HunteCrossley model generally captured the experimental contact times well with a mean difference between experimental and model contact times of $8.3 \%$. The mean difference between the KelvineVoigt model and experimental contact times was 7.6\%, while the corresponding mean difference for the coefficient of restitution was $13.1 \%$. Overall, the modified KelvineVoigt model predicted the parameters of contact time and coefficient of restitution well. Contact time and coefficient of restitution prediction in this linear model were not particularly sensitive to the strain rate.
\end{abstract}

\section{Keywords:}

Phenomenological and physical dynamic, models, HunteCrossley, KelvineVoig,t Modulus of elasticity, Hertzian contact stiffness.

\section{Introduction}

Hurling is one of the national Irish sports governed by the Gaelic Athletic Association (GAA) inwhich a ball is struck by awooden stick known as a hurley. The ball, known as a sliotar, consists of a leather skin and a solid core. The sliotar has a standardised mass of $90 \_5 \mathrm{~g}$ and diameter of $67 \_2 \mathrm{~mm}$ (Gaelic Athletic Association, 2008) [1].The materials fromwhich the cores are manufactured can be divided into two categories: the more traditional cork wrapped in a yarn winding and the more modern polymer foam core. It has been identified that the response of the ball when struck by a hurley is dominated by the behaviour of the core material (Gaelic Athletic Association, 2004) [2]. This gives rise to the question: how does one manufacture a ball such that it exhibits a specific performance? This question remains unanswered as, until recently, the exact characteristics of the dynamic response had not been sufficiently studied or modelled to allow for sliotar standardisation. This led the GAA to commission a study to examine the dynamic behaviour of the ball core (Collins et al., 2011) [3]. The aim of this previous study was characterise the performance of different core materials in terms of significant impact parameters. To obtain objective and repeatable impact parameters, a standard impact configuration was examined rather than actual hurley/sliotar contact. The chosen configuration was the direct, normal impact of a non-rotating ball core 
against a static, rigidly-mounted fixed steel plate. The platewas assumed to be infinitely stiff compared to the ball core so that no momentum was exchanged during the impact. Such an impact configuration allowed for intrinsic measurement of the mechanical response of the ball material. Such a methodology is consistent with similar studies of other types of sports ball including tennis, cricket and golf balls (e.g., Carré et al., 2004; Cheng et al., 2008; Cross, 1999; Fuss, 2008) [4e7].Players' perception of ball performance features subjective attributes such as liveliness and 'feel'. Such non-scientific measures can be quantified in terms of energy dissipation and hardness of the ball, which, in theory, may be related to impact parameters such as the coefficient of restitution (ratio of ball speeds after and before impact), impact duration (contact time), the maximum impact force and deformation of the ball.

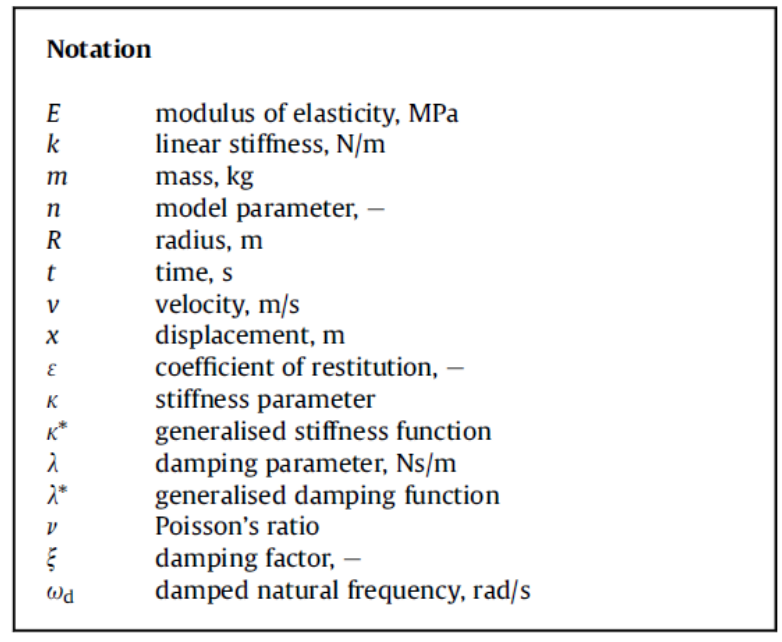

For example, a contact time that is excessively short usually corresponds to a ball being considered too lively or 'bouncy'. Insufficient deformation generally implies that the ball is excessively stiff which in turn indicates that an inadvertent collision between the ball and player could result in a serious injury. Conversely, excessive ball deformation can affect the durability of the sliotar. The coefficient of restitution is the only impact parameter that is regulated at present; it must lie between 0.522 and 0.576 when measured from a drop height of $1.8 \mathrm{~m}$. This corresponds to an impact speed of around $6 \mathrm{~m} / \mathrm{s}$ and is the only speed at which this coefficient is specified. A coefficient of restitution outside this range generally means that the ball will be either too 'dead' or too uncontrollable if the coefficient is too low or too high, respectively. As $6 \mathrm{~m} / \mathrm{s}$ is considerably lower than actual impact speeds during play, which are typically in the range of 10-40 m/s, the current approval test cannot account for variations between approved balls that may become apparent at higher speeds.

This test method is similar to the regulatory testing used for other ball types, e.g., the International Tennis Federation employs a 100 inch drop test for tennis balls. The inadequacy of this means of regulating coefficient of restitution has been recognised in recent studies (Carré et al., 2004; Cross, 1999) [4,6]; this test does not represent the full range of conditions experienced in play which is problematic due to the non-linear dependence of coefficient of restitution on impact speed.There are many continuous contact dynamic models in literature that relate force and deformation during impact (Gilardi and Sharf, 2002) [8]. Such models have been applied successfully to represent the impact response of other types of solid and 
hollow sports balls (e.g., Cheng et al., 2008; Cochran, 2002; Goodwill and Haake, 2004) $[5,9,10]$. These models generally insert some combination of conceptual springs and dashpots at the contact point between bodies. The dashpots dissipate energy while the springs provide the required elastic behaviour. A more detailed review of mathematical models in the context of sports ball impact has been presented by Collins (2011) [11]. FE models have been used to model ball impacts (e.g., Cheng et al., 2008; Tanaka et al., 2006)[5,12]. Primary attractions of spring-dashpot based numerical models are that they offer quick and efficient analysis, and that model parameters (i.e., spring stiffness and dashpot dissipation)pertain directly to impact parameters (i.e., material stiffness and energy dissipation). In this paper, the objective was to develop the first model for the sliotar core impact behaviour.

\section{Theory}

\subsection{Modelling approach}

Many of the impact models which are in use can be summarised as the sum of three terms: an inertia term, a damping term and a stiffness term. The impact between the ball and the rigid fixed flat plate may be treated as a single degree of freedom problem. The damping and stiffness terms are usually proportional to impact velocity and displacement, respectively, and can be expressed as:

$$
m x^{\prime \prime}(t)+\lambda^{*}\left(x, x^{\prime}\right) x^{\prime}(t)+\kappa^{*}\left(x, x^{\prime}\right) x(t)=0
$$

$1^{*}$ and $\mathrm{k}^{*}$ represent generalised damping and stiffness functions, respectively, which may in turn be functions of impact velocity and displacement. One common approach in the literature is to restrict these functions to having a power law dependence on displacement.

$$
\lambda^{*}\left(x, x^{\prime}\right)=\lambda x^{n_{1}} \quad \kappa^{*}\left(x, x^{\prime}\right)=\kappa x^{n_{2}}
$$

The possible strain rate dependence of $\mathrm{k}$ is thus neglected and the fundamental impact model becomes:

$$
m x^{\prime \prime}(t)+\lambda x^{n_{1}} x^{\prime}(t)+\kappa x^{n_{2}} x(t)=0
$$

Three models of ball impact are commonly seen in sports literature, all of which are special cases of Eq. (3) for particular choices of $n 1$ and $n 2$ :

\section{1) Linear KelvineVoigt Model $\left(\mathrm{n}_{1}=\mathrm{n}_{2}=0\right)$}

The linear KelvineVoigt model is presented in Eq. (4). Parameter 1 corresponds to the linear viscous damping coefficient, commonly denoted by $\mathrm{c}$, while $\mathrm{k}$ corresponds to the linear spring stiffness, $\mathrm{k}$. The damping term in the KelvineVoigt model is directly proportional to the velocity. This linear model was applied to golf balls (Cochran,1998) [13]. This modelwas implemented in this work due to its relatively simple nature, the fact that the parameters of the model have a physically-meaningful interpretation and that it can be solved analytically as a function of time. The linear KelvineVoigt model is determined by substituting $\mathrm{n}_{1}=\mathrm{n}_{2}=$ 0 into Eq. (3):

$$
m x^{\prime \prime}(t)+\lambda x^{\prime}(t)+\kappa x(t)=0
$$


For this model, the parameters 1 and $\mathrm{k}$ are also taken as constants (for all displacements and speeds); however, an $\mathrm{x} 0.5$ term is included in both the damping and stiffness terms to account for

the Hertzian nature of the contact. Thus, the damping and stiffness terms both vary nonlinearly. This approach was considered by Cochran (2002) [9] in developing simple onedimensional models of golf ball impacts. This model was not implemented for comparison in this work as although it is a simple model, it presented no clear advantage over results that would be alculated from the modified KelvineVoight model and the HunteCrossley model which were implemented. The linear Hertz impact model is determined by substituting $\mathrm{n}_{1}=\mathrm{n}_{2}$ $=0.5$ into Eq. (3):

$$
m x^{\prime \prime}(t)+\lambda x^{0.5} x^{\prime}(t)+\kappa x^{1.5}(t)=0
$$

3) HunteCrossley Model $\left(n=n_{1}=n_{2}+1\right)$

This differential equation was originally developed by Hunt and Crossley (1975) [14]. It permits impacts between bodies to be modelled in a relatively straightforward manner using a small number of model parameters, and yields results which are more consistent with experimental findings than the KelvineVoigt model (Gilardi and Sharf, 2002) [8]. The HunteCrossley model was selected as the main analytical tool for this study as an implicitformulation between velocity and displacement can be determined from this model. The HunteCrossley model is determined by substituting $n=n_{1}=n_{2}+1$ into Eq. (3):

$$
m x^{\prime \prime}(t)+\lambda x^{n} x^{\prime}(t)+\kappa x^{n}(t)=0
$$

\subsection{HunteCrossley model}

It is not possible to obtain an analytical solution for impact displacement, velocity or acceleration as a function of time using Eq. (6). However, Marhefka and Orin (1996) [15] demonstrated an implicit relationship formulation between velocity and displacement as:

$$
\frac{\kappa}{m} \ln \left|-\frac{\kappa}{m}-\frac{\lambda}{m} v\right|-\frac{\lambda v}{m}=\frac{\lambda^{2}}{m^{2}(n+1)} x^{n+1}-\frac{\lambda v_{0}}{m}+\frac{\kappa}{m} \ln \left|-\frac{\kappa}{m}-\frac{\lambda}{m} v_{0}\right|
$$

where $v_{0}$ is the initial impact velocity and other variables are as already noted. Eq. (8) presents a relationship for calculating the linear viscous damping coefficient developed by Marhefka and Orin, which was derived with the assumption of a low impact velocity. This equation relates the damping and stiffness parameters of the model ( $\lambda$ and $k$ ) to two measurable experimental quantities: the initial impact velocity, $\mathrm{v}_{0}$, and the coefficient of restitution, $\varepsilon$.

$$
\lambda=\frac{3 \kappa(1-\varepsilon)}{2 v_{0}}
$$


Hunt and Crossley (1975) suggested that the most appropriate value for $\mathrm{n}$ depends on the geometry of the contacting bodies [14].For this situation, the magnitude of $n$ must reflect the fundamental geometry of a sphere impacting a rigid fixed flat plate. Therefore, the Hertzian value of 1.5 was chosen [14]. Substituting this in for $n$ in Eq. (6), gives the following HunteCrossley impact model:

$$
m x^{\prime \prime}(t)+\lambda x^{1.5} x^{\prime}(t)+\kappa x^{1.5}(t)=0
$$

The Hunte-Crossley model requires four parameter values; $\mathrm{m}, \lambda, \mathrm{k}$ and $\mathrm{n}$. Since the mass of the ball core is known a priori and the magnitude of $\mathrm{n}$ is fixed by the geometry of the contact, only two parameters remain to be obtained: $\lambda$ and $\mathrm{k}$. The magnitudes of these two parameters are usefully found from analysis of experimental data obtained from impact tests of the ball cores. The stiffness parameter, $\mathrm{k}$, is obtained from data-fitting the force deflection curves recorded experimentally for physical impacts of the ball cores with a steel plate, i.e., the deviations between the experimental force deflection data points and the model predictions were minimised by varying $\mathrm{k}$. For each trial value of $\mathrm{k}$, a corresponding value was quantified for the damping parameter, $\lambda$, using Eq. (8) and measurements of the initial impact velocity and coefficient of restitution. Hence, $\lambda$ is velocity dependent and has a value that is specific both to the ball type and to the incident impact speed. Thus, the stiffness parameter is an empirically-fitted factor in the model as the damping term is a function of $\mathrm{k}$ and two measurable impact quantities.

It is possible to relate $\mathrm{k}$ to the asymptotic Hertzian stiffness parameter, ${ }^{\mathrm{k}} \mathrm{H}$. As the strain rate approaches zero (i.e., at lower impact speeds), the damping term in Eq. (9) becomes negligible Furthermore, as the impact displacement or strain also approaches zero, then the material stresses train relationship should also be approximately linear. For this situation, the generalised Hertzian stiffness of the ball, ${ }^{\mathrm{k}} \mathrm{H}$, can be related to the modulus of elasticity of the core, E, as follows:

$$
\operatorname{Lim} \kappa \rightarrow \kappa_{\mathrm{H}}=\frac{2 E}{3\left(1-v^{2}\right)} \sqrt{R} \text { as } x^{\prime} \rightarrow 0 \text { and } x \rightarrow 0
$$

where $\mathrm{n}$ is Poisson's ratio and $\mathrm{R}$ is the radius of the core (Young,2001) [16]. The Hertzian stiffness is the stiffness that the ball would exhibit under quasistatic compression. For typical physical impacts (impact speed of approximately $10 \mathrm{~m} / \mathrm{s}$ and where maximum ball deformation at impact is large) the stiffness exhibited by the ball, $\mathrm{k}$, will be considerably different due to non-linearity in the material response at high strain and to strain-rate effects. However, Eq. (10) supplies some physical interpretation (although not a complete explanation) of the physical basis of $\mathrm{k}$.

\subsection{Equivalent Kelvine-Voigt model}

The Kelvine-Voigt model (or any model based on it) has a number of advantages: it is simple, the parameters of the model have a physically-meaningful interpretation and it can be solved analytically for impact velocity and displacement as a function of time. Its main 
weakness is that some of its predictions are both logically untenable and conflict with experimental measurements. More specifically, the Kelvine-Voigt model predicts that contact forces at the beginning of the impact are discontinuous (due to the damping term being proportional to velocity alone), that coefficients of restitution do not vary impact velocity (which is inaccurate, as COR has been shown experimentally to decrease with impact speed) and that small attractive force terms appear directly prior to the separation of the bodies (when from physical reasoning, if the body has expanded back to its original dimensions, no net force should act upon it). Such deficiencies were also observed by Haake et al.(2003) and Goodwill and Haake $(2001,2004)$ [10,17,18]. None-theless,comparing the Hunte-Crossley model predictions to an analogous Kelvine-Voigt model can facilitate understanding of the impact process.

Impacts of the sliotar core against a rigid surface may be shown to be always under-damped. Using the impulse-response technique, the solution to the governing differential equation for impact displacement (Eq. (4)) is:

$$
x(t)=\frac{v_{0}}{\omega_{\mathrm{d}}} e^{-\xi \omega_{\mathrm{n}} t} \sin \left(\omega_{\mathrm{d}} t\right) \quad \text { where } \omega_{\mathrm{d}}=\omega_{\mathrm{n}} \sqrt{\left|1-\xi^{2}\right|}
$$

where, $\omega_{\mathrm{n}}$ is the natural frequency, $\omega_{\mathrm{d}}$ is the damped natural frequency, and $\xi$ is the damping factor. Hence, the duration of impact or contact time, $t_{c}$, can be quantified in terms of the mass, damping and stiffness coefficients as:

$$
t_{\mathrm{c}}=\frac{\pi}{\omega_{\mathrm{d}}}=\frac{2 \pi m}{\sqrt{4 m k-\lambda^{2}}}
$$

By differentiating the impact displacement with respect to time to find the impact velocity and comparing the magnitudes of velocity at the end of the contact time (the rebound velocity) to the initial velocity, $\mathrm{v}_{0}$ (the approach velocity), a corresponding expression for the coefficient of restitution, $\varepsilon$, is obtained:

$$
\varepsilon=e^{\frac{-\lambda \pi}{\sqrt{4 m k-\lambda^{2}}}}
$$

Based on the KelvineVoigt model, Eqs. (12) and (13) predict the magnitudes of defining impact quantities in terms of intrinsic system constants.

By selecting a representative fixed displacement, $\mathrm{xR}$, for each impact condition, and substituting into Eq. (9), the nonlinear Hunt-Crossley model is linearly approximated as:

$$
m x^{\prime \prime}(t)+\lambda x_{\mathrm{R}}^{1.5} x^{\prime}(t)+\kappa x_{\mathrm{R}}^{0.5} x(t)=0
$$


Hence, an equivalent viscous damping coefficient and equivalent linear stiffness can be defined as:

$$
\lambda_{\mathrm{eq}}=\lambda x_{\mathrm{R}}^{1.5} \text { and } k_{\mathrm{eq}}=\kappa x_{\mathrm{R}}^{0.5}
$$

An important parameter to quantify the linear impact model is the dimensionless damping factor, $\mathrm{x}$, which relates mass, and equivalent linear stiffness and equivalent viscous damping coefficient:

$$
\zeta=\frac{\lambda_{\mathrm{eq}}}{2 \sqrt{k_{\mathrm{eq}} m}}
$$

\section{Materials and methods}

\subsection{Sliotar cores}

Four sliotar cores were available for this work, which were labelled as ball types AeD. Table 1 quantifies the dimensions, masses and compositions of these cores. The experimental programme consisted of tests to measure the modulus of elasticity of each ball core, to assess its response under quasi-static loading and to measure its performance at full impact conditions. The results for one sample of each ball type are presented in this paper.

\section{Table 1}

Diameters, masses and compositions of each sliotar core used in this study.

\begin{tabular}{llll}
\hline Ball ID & Diameter $(\mathrm{mm})$ & Mass $(\mathrm{g})$ & Material mass composition \\
\hline A & $66.2 \pm 0.1$ & $89.6 \pm 0.4$ & $100 \%$ polyurethane-based polymer \\
B & $66.8 \pm 0.2$ & $89.9 \pm 3.1$ & $100 \%$ polyurethane-based polymer \\
C & $65.6 \pm 0.1$ & $89.1 \pm 0.9$ & Cork (81\%), yarn (19\%) \\
D & $68.2 \pm 0.4$ & $83.1 \pm 1.3$ & Cork (38\%), polyester (38\%), yarn (24\%) \\
\hline
\end{tabular}

\subsection{Hertz stiffness parameter tests}

In order to measure the modulus of elasticity of each material (polyurethane for ball A and B; cork for ball $\mathrm{C}$ and $\mathrm{D}$ ), blocks (in the shape of rectangular cuboids) were cut centrally from randomly sampled ball cores and subjected to quasi-static compression using a Stable Micro Systems TA.HD plus texture analyser (Stable Micro Systems Ltd., Godalming, Surrey, UK) with a $250 \mathrm{~kg}(2.452 \mathrm{kN})$ load cell. Rectangular blocks were preferred to cylindrical samples for this work as each sample could be cut precisely from the spherical cores to have dimensions which exhibited little variation along the direction of loading, which would be considerably more difficult to achieve with cylindrical samples.

Each block had a height of approximately $31 \mathrm{~mm}$, measured parallel to the direction of loading, and cross-sectional dimensions of $22 \mathrm{~mm}$ by $24 \mathrm{~mm}$. The contact surfaces of the texture analyser were made of stainless steel with a polished surface finish to reduce the coefficient of friction. Each block was compressed at $10 \mu \mathrm{m} / \mathrm{s}$ until a deflection of $10 \mathrm{~mm}$ was attained. Blocks were tested once before being discarded, and the Mullins effect was neglected. The force and deflection data obtained were used to calculate the modulus of elasticity. Poisson's ratio was taken as 0.4 for all samples. Intact sliotar cores of each type 
were compressed similarly to maximum deflections of $16 \mathrm{~mm}$ to determine the force deflection behaviour of these spherical bodies. The force $(\mathrm{F})$ and deflection $(\mathrm{x})$ data for each ball from these tests were fitted to the equation presented in Eq. (17), with the Hertzian stiffness of the ball, $\mathrm{kH}$, and with the exponent fixed at 1.5:

$$
F(x)=\kappa_{\mathrm{H}} x^{1.5}
$$

The magnitude of the constant term (the Hertzian stiffness parameter, $\mathrm{kH}$ ), was found by least-squares data fitting using the curve fitting toolbox in MATLAB (v.7.9.0, The MathWorks, Natick, MA, USA). Thus the Hertzian stiffness parameter was determined using two different methods, one from engineering constants (Eq.(10)) and phenomenologically from impact results, and the results compared.

\subsection{Full impact experiments}

The four sliotar core types were subjected to rigid-body impacts in the velocity range of 5-25 $\mathrm{m} / \mathrm{s}(15-90 \mathrm{~km} / \mathrm{h})$ using a test system specifically commissioned to characterise the impact response of sports balls. Ball speed before and after impact was acquired using high-speed imaging at $4000 \mathrm{fps}$, with the data being extracted using an image processing algorithm (Collins et al., 2009) [19]. Viscoelastic properties of the cores were acquired from forceetime data obtained using a load-cell integrated within the fixed rigid impact plate. Calibration of this system showed all force and impulse measurements to be within _ $10 \mathrm{~N}$ and _3\% of actual values respectively [20]. The experimentally-measured deflections corresponded to the centre-of-mass displacements of the balls, as calculated from the double time integral of the force data divided by ball mass. Further details of these experiments are provided in a previous publication (Collins et al., 2010) [20]. Fig. 1 illustrates the deformation of a ball core upon impact with the fixed rigid steel impact plate.

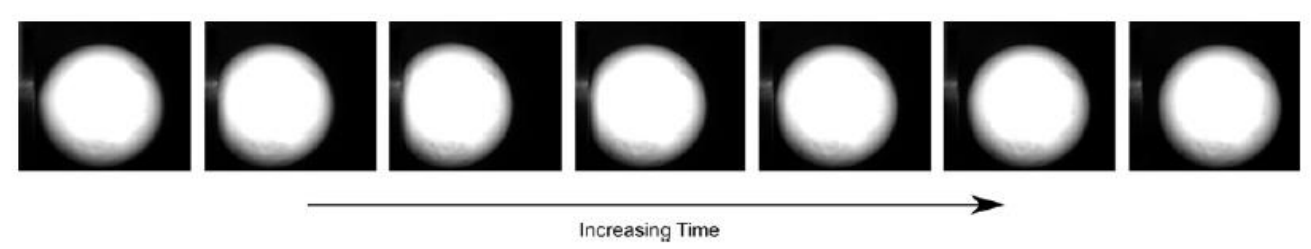

Fig. 1. Frames extracted from the video of an impact test of ball core $A$ at $15 \mathrm{~m} / \mathrm{s}$, in which the time increment between adjacent frames is $0.5 \mathrm{~ms}$.

\section{Results and discussion}

\subsection{Modulus of elasticity and Hertzian contact stiffness}

Fig. 2 compares the force versus deflection curves for experimental quasi-static compression tests of intact sliotar cores with the corresponding power law fitted to the data using the Hertzian exponent value of 1.5, using Eq. (17). These fitted power law curves are shown as red dashed lines on Fig. 2. The moduli of elasticity and Hertzian stiffness parameters are directly proportional, as shown by Eq. (10). The data is shown over the range from 0 to 2.5 $\mathrm{mm}$, as Eq. (10) is valid only for small displacements. The power law fitted the data extremely well for both of the modern polymer cores $\left(\mathrm{R}^{2}\right.$ values in excess of $\left.99 \%\right)$ and the fit was also good for cores $\mathrm{C}$ and $\mathrm{D}$ (R2 values were still above $96 \%$ ). 

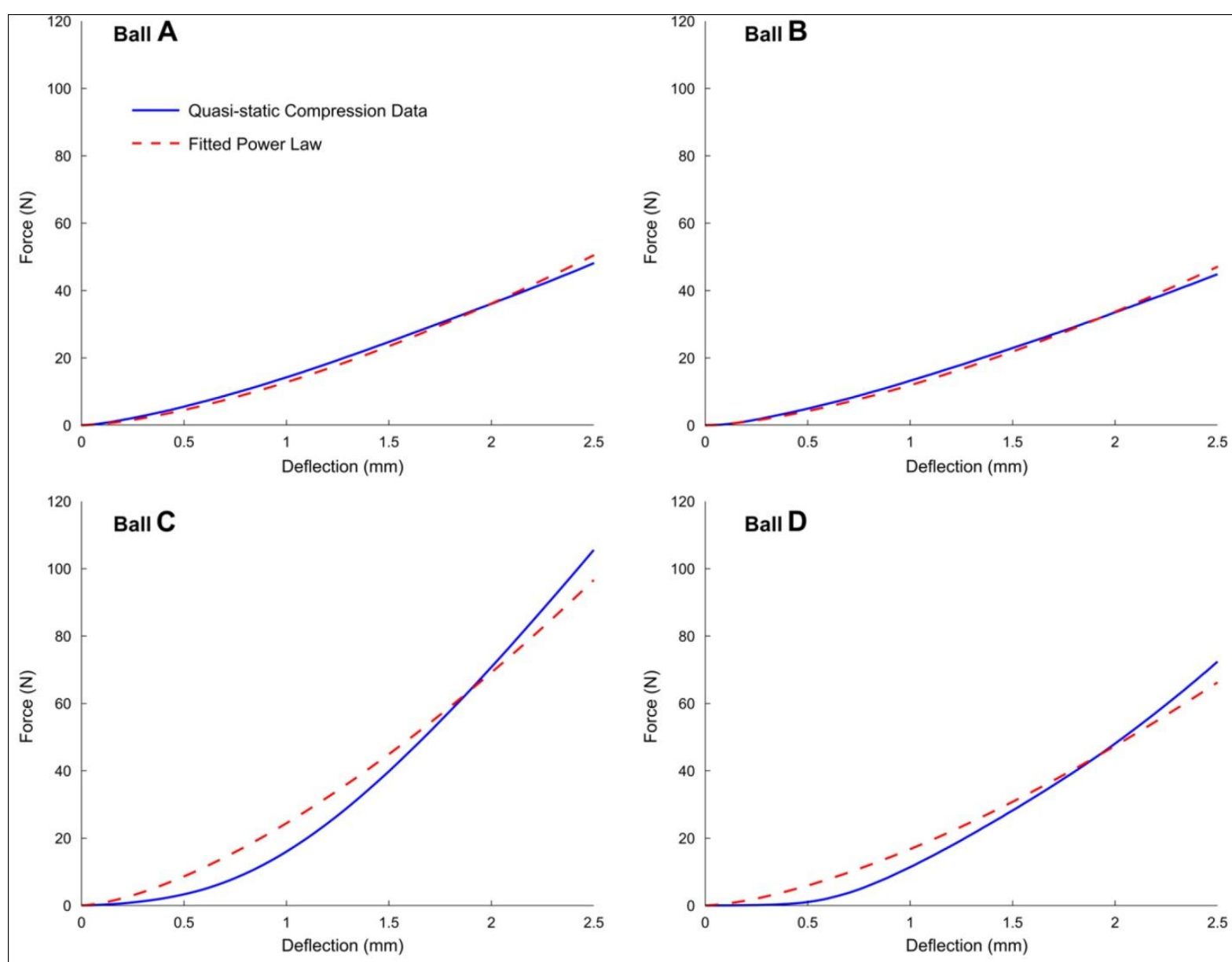

Fig. 2. Force $(\mathrm{N})$ versus deflection $(\mathrm{mm})$ data for quasi-static compression of four ball cores. The red dashed line on each plot indicates the power law fitted to the initial $1 \mathrm{~mm}$ of deflection in which the behaviour was approximately linear. (For interpretation of the references to colour in this figure legend, the reader is referred to the web version of this article.)

The good agreement between theory and experiment indicates that, at least at low load levels and strain rates, the contact mechanics of the ball cores were described well by Hertzian theory.As discussed in Section 3.2, the moduli of elasticity were calculated from quasi-static compression tests of regular blocks cut from each ball core. These results are shown in Table 2, along with the corresponding predicted Hertzian stiffness parameters $(\mathrm{kH}$, calculated from Eq. (10)).

The cork-based cores $\mathrm{C}$ and $\mathrm{D}$ had significantly higher moduli of elasticity than the polyurethane-based cores of $\mathrm{A}$ and $\mathrm{B}$. Core $\mathrm{C}$ had the largest modulus of elasticity reflecting the fact that its composition was $81 \%$ cork while that of core D was only $38 \%$ cork. This in turn meant that the corresponding theoretical Hertzian stiffness, $\mathrm{kH}$, was greater for these cores. For the polyurethanebased cores, there was reasonable agreement between the theoretical Hertzian stiffness parameter and the value measured from the experimental quasistatic tests, while the correspondence was considerably poorer for the cork-based cores.

However, core $\mathrm{C}$ was predicted as the stiffest in both approaches. There were two main reasons for the disparity between the theoretical and experimental Hertzian stiffness parameters. Firstly, the power law regressions in Fig. 2 were fitted using a $2 \mathrm{~mm}$ range of displacement $(0.5 \mathrm{e} 2.5 \mathrm{~mm})$; if the regression were performed using a different range of displacement, this would significantly affect the results. 
Table 2

Moduli of elasticity for blocks cut from the four ball cores, corresponding calculated Hertzian stiffness parameters and the experimental equivalents obtained from the quasi-static compression of each intact ball core.

\begin{tabular}{lcll}
\hline Ball ID & $\begin{array}{l}\text { Modulus of } \\
\text { elasticity, } E(\mathrm{MPa})\end{array}$ & $\begin{array}{l}\text { Theoretical Hertzian } \\
\text { stiffness parameter } \\
\left(\mathrm{N} / \mathrm{m}^{1.5} \times 10^{5}\right)\end{array}$ & $\begin{array}{l}\text { Experimental } \\
\text { Hertzian stiffness } \\
\text { parameter }\left(\mathrm{N} / \mathrm{m}^{1.5} \times 10^{5}\right)\end{array}$ \\
\hline A & 1.94 & 2.81 & 4.053 \\
B & 3.47 & 5.03 & 3.783 \\
C & 11.7 & 17.0 & 7.731 \\
D & 9.3 & 13.5 & 5.316 \\
\hline
\end{tabular}

In general, avoiding the non-linearity portions at the start and end sections of these curves provided better results. Secondly, initial part of the stress-strain curves showed large deviations from linearity, which may be due to effects such as buckling, collapse or consolidation of the core microstructure. As for the power law regressions, the calculated moduli of elasticity varied depending on the region defined to be linear.

\subsection{Non-linear impact parameters}

An algorithm was written in MATLAB to find the optimum value of $\mathrm{k}$ for each core to fit the Hunte-Crossley model to the impact data. The damping parameter, $\lambda$, was calculated from $\mathrm{Eq}(8)$ for each ball and nominal impact speed. Table 3 summarises the magnitudes of $\mathrm{k}$ and $\lambda$ found for each ball core. The Hunte-Crossley stiffness parameter for the ball cores, $\mathrm{k}$, did not vary greatly between the four different ball types. This was the case even though the moduli of elasticity of the ball cores showed significant differences (Table 2) and the Hertzian stiffness parameters are proportional to these moduli (Eq. (10)). Ball $\mathrm{C}$ had the largest value for $\mathrm{k}$ which may reflect the fact that it was the ball with the largest modulus of elasticity. The damping parameter, $\lambda$, was particularly sensitive to impact speed, decreasing with higher speeds. Again, the magnitude of 1 for ball $\mathrm{C}$ was distinct from the other three core materials. As ball $\mathrm{C}$ had the largest stiffness and damping parameters, it would be expected to develop the largest contact forces during impact.

\section{Table 3}

Parameters calculated for the Hunt and Crossley model.

\begin{tabular}{llllr}
\hline Ball ID & $\kappa\left(\mathrm{N} / \mathrm{m}^{1.5} \times 10^{6}\right)$ & \multicolumn{3}{l}{$\lambda\left(\mathrm{Ns} / \mathrm{m}^{2.5} \times 10^{4}\right)$} \\
\cline { 3 - 5 } & & $5 \mathrm{~m} / \mathrm{s}$ & $15 \mathrm{~m} / \mathrm{s}$ & $25 \mathrm{~m} / \mathrm{s}$ \\
\hline A & 3.50 & 37.69 & 17.63 & 11.64 \\
B & 2.85 & 31.07 & 13.86 & 9.20 \\
C & 5.65 & 60.63 & 28.13 & 17.73 \\
D & 3.70 & 36.38 & 17.85 & 11.75 \\
\hline
\end{tabular}




\subsection{Non-linear analysis of impact response}

Fig. 3 shows plots of force versus deflection at the three impact speeds for each ball type as recorded experimentally and as predicted by the model, while Fig. 4 shows equivalent plots of deflection versus time. Agreement between experiment and model was generally very good. These two figures show only one set of experimental data (in blue) for comparison purposes. In all cases, the set chosen for comparison was representative of all the data recorded. The maximum contact force was relatively insensitive to ball type, although ball C was again most distinct from the others. Unsurprisingly, the maximum contact force was very sensitive to impact speed; typically it varied from $750 \mathrm{~N}$ at $5 \mathrm{~m} / \mathrm{s}$ impact to $2500 \mathrm{~N}$ at $15 \mathrm{~m} / \mathrm{s}$ and $4500 \mathrm{~N}$ at $25 \mathrm{~m} / \mathrm{s}$. The maximum impact deflection also varied considerably with impact speed: $3 \mathrm{~mm}$ at $5 \mathrm{~m} / \mathrm{s}$ impact, $6 \mathrm{~mm}$ at $15 \mathrm{~m} / \mathrm{s}$ and $10 \mathrm{~mm}$ at $25 \mathrm{~m} / \mathrm{s}$.
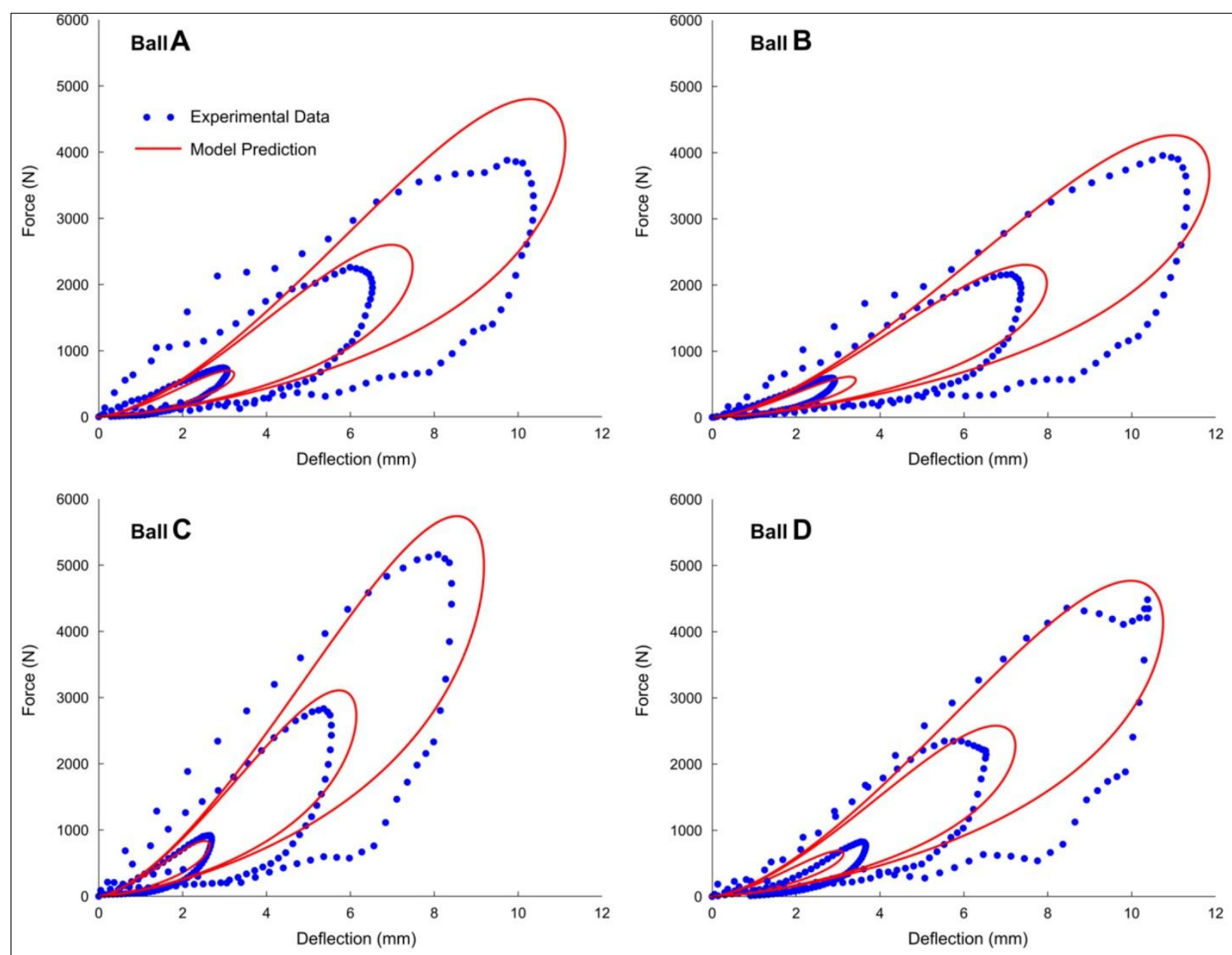

Fig. 3. Comparison of the experimental impact force $(\mathrm{N})$ versus deflection $(\mathrm{mm})$ data with the results predicted by the Hunt-Crossley model for the four cores at impact speeds of $5 \mathrm{~m} / \mathrm{s}, 15 \mathrm{~m} / \mathrm{s}$ and $25 \mathrm{~m} / \mathrm{s}$.

The model had a slight tendency to over-predict both the maximum force and maximum deflection for each ball type at each impact speed.Overall, ball $\mathrm{C}$ gave the largest maximum impact force but the smallest maximum displacement of the four cores tested; its short and hard contact reflected the large stiffness and damping forces that were generated in impact (Fig. 5).The contact times are shown on the horizontal axes of Fig. 4. 

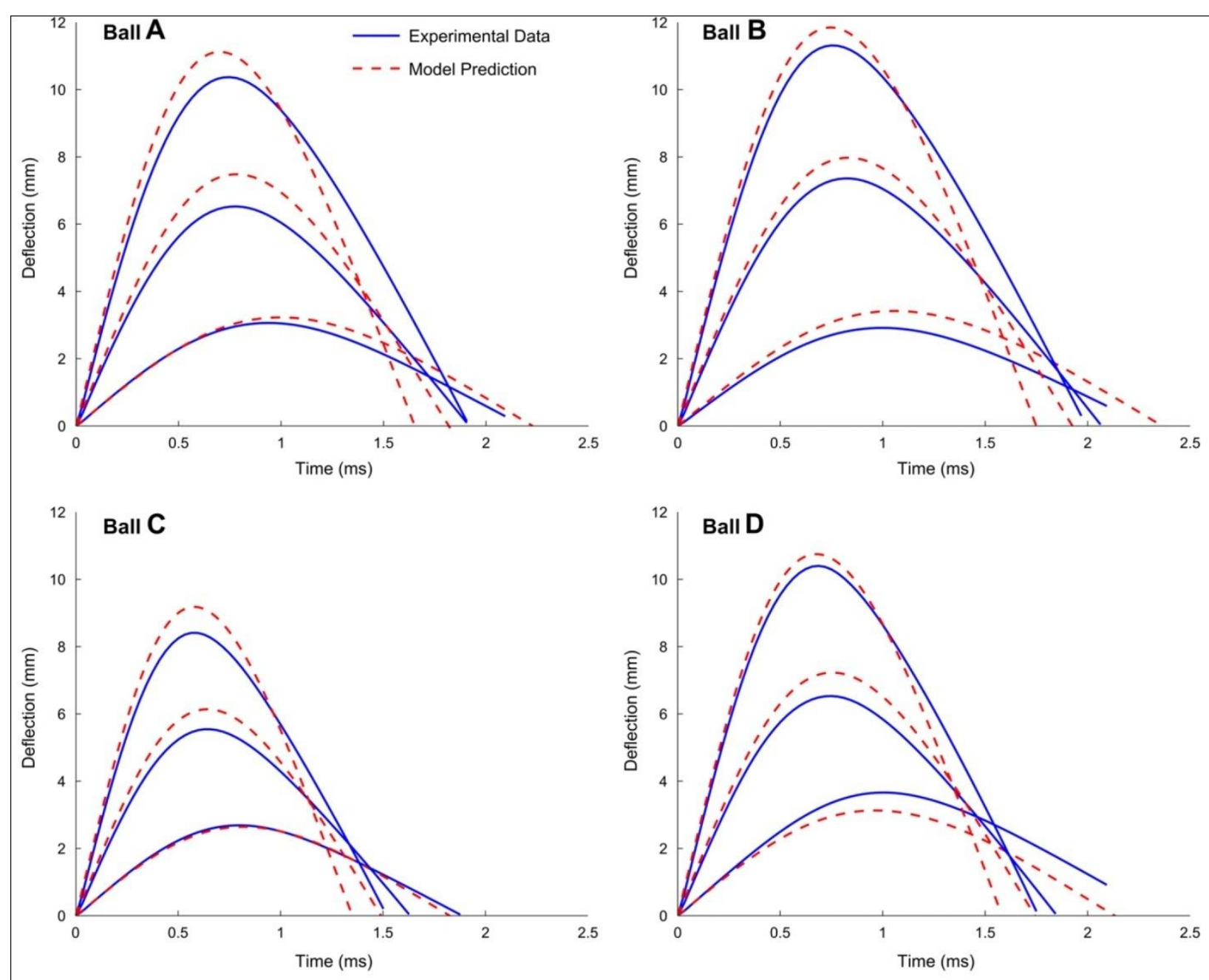

Fig. 4. Comparison of the experimental impact deflections $(\mathrm{mm})$ versus time $(\mathrm{ms})$ with the results predicted by the Hunt-Crossley model for the four cores at impact speeds of $5 \mathrm{~m} / \mathrm{s}, 15 \mathrm{~m} / \mathrm{s}$ and $25 \mathrm{~m} / \mathrm{s}$.

The contact times recorded experimentally decreased with increasing impact velocity although its sensitivity to speed was not substantial.

The model generally captured the experimental contact times well, although a tendency to under-predict the experimental data was apparent for nine of the twelve points shown. However, the maximum difference between the experimental and model contact times was low: only $13.5 \%$ of the experimental data, while the mean difference was $8.3 \%$. The model contact time was quite sensitive to changes in the impact speed: average model contact times were $2.14 \mathrm{~ms}$ at $5 \mathrm{~m} / \mathrm{s}$ which reduced to $1.58 \mathrm{~ms}$ at $25 \mathrm{~m} / \mathrm{s}$.

\subsection{Equivalent linear system analysis}

By selecting a representative or characteristic value for impact deflection, $x R$, an equivalent viscous damping coefficient, equivalent linear stiffness and dimensionless damping factor can be determined for each ball type using Eqs. (15) and (16). This representative deflection was set as the mean of the deflections recorded during an impact. Table 4 summarises the representative deflections measured and the results calculated for the equivalent linear dynamic model, where the necessary values of $\mathrm{k}$ and 1 are taken from Table 3. 


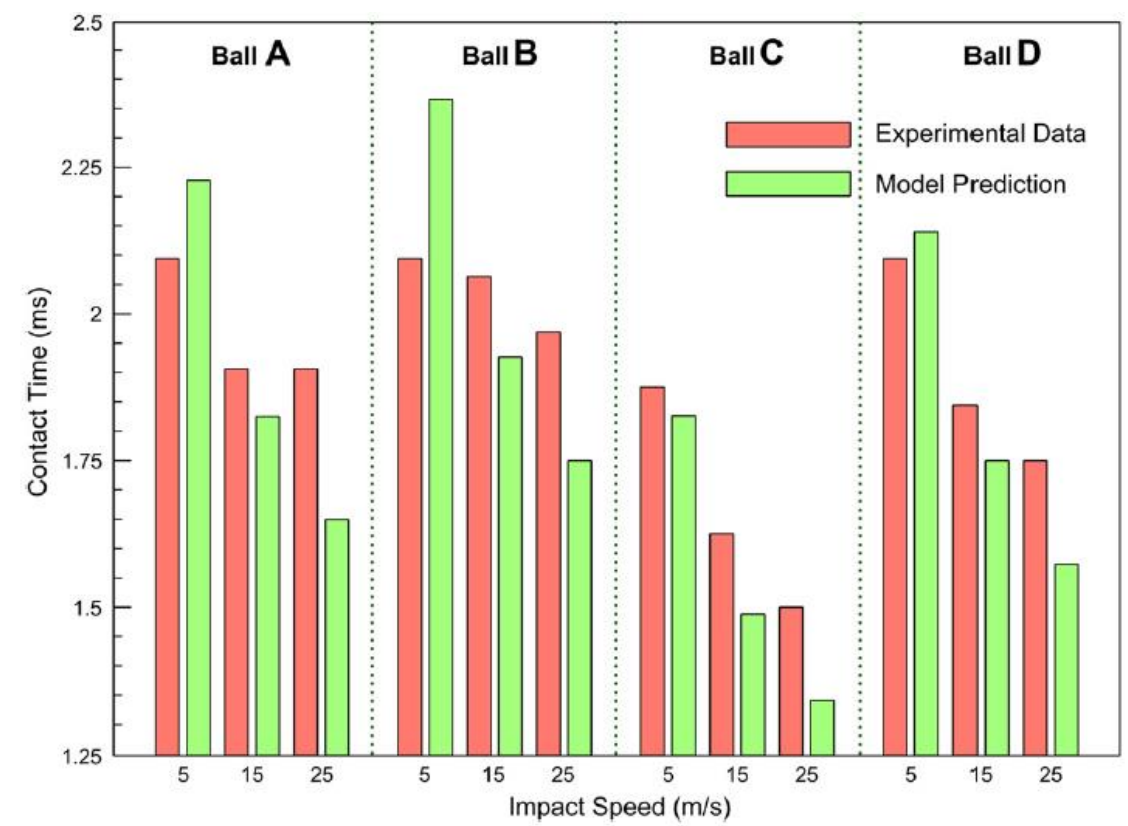

Fig. 5. Bar charts comparing the contact times (ms) measured experimentally for impact tests of four ball cores at three speeds with the predictions made by the Hunt-Crossley model.

The representative deflection was quite dependent on impact speed, typically rising from less than $2 \mathrm{~mm}$ at $5 \mathrm{~m} / \mathrm{s}$ to over $6 \mathrm{~mm}$ at $25 \mathrm{~m} / \mathrm{s}$, but relatively insensitive to the ball type. The calculated equivalent damping and stiffness coefficients increased with impact speed for each ball. As expected, $\lambda_{\text {eq }}$ and $\mathrm{k}_{\mathrm{eq}}$ were highest for ball $\mathrm{C}$ at each impact speed (except $\lambda_{\mathrm{eq}}$ at 5 $\mathrm{m} / \mathrm{s})$.

Table 4

Results calculated for the equivalent linear dynamic model.

\begin{tabular}{lllll}
\hline $\begin{array}{l}\text { Impact } \\
\text { speed } \\
(\mathrm{m} / \mathrm{s})\end{array}$ & $\begin{array}{l}\text { Representative } \\
\text { deflection, }\end{array}$ & $\begin{array}{l}\text { Viscous damping } \\
\text { coefficient, } \\
c_{\mathrm{R}}(\mathrm{mm})\end{array}$ & $\begin{array}{l}\text { Linear stiffness, } \\
k_{\mathrm{eq}}(\mathrm{N} / \mathrm{m})\end{array}$ & $\begin{array}{l}\text { Dimensionless } \\
\text { damping factor, } \xi\end{array}$ \\
\hline Ball $\mathrm{A}$ & & & & \\
5 & 1.93 & 31.95 & 1.54 & 0.136 \\
15 & 3.97 & 44.05 & 2.20 & 0.156 \\
25 & 6.30 & 58.13 & 2.78 & 0.184 \\
Ball B & & & & \\
5 & 1.91 & 25.93 & 1.25 & 0.122 \\
15 & 4.43 & 40.88 & 1.90 & 0.156 \\
25 & 6.85 & 52.14 & 2.36 & 0.179 \\
Ball C & & & & \\
5 & 1.62 & 39.51 & 2.27 & 0.139 \\
15 & 3.32 & 53.73 & 3.25 & 0.158 \\
25 & 5.06 & 63.78 & 4.02 & 0.169 \\
Ball D & & & & \\
5 & 2.40 & 42.68 & 1.81 & 0.174 \\
15 & 3.93 & 44.02 & 2.32 & 0.159 \\
25 & 6.22 & 57.71 & 2.92 & 0.185 \\
\hline
\end{tabular}


It is apparent that cores made of traditional materials incorporating cork have greater viscous damping and are stiffer than modern polyurethane-based polymer cores. The dimensionless damping factor was relatively invariant for all ball types and had a moderate tendency to increase with larger impact speeds.

Using these magnitudes of equivalent linear stiffness and damping, predictions of both the contact time and the coefficient of restitution were made, using Eqs. (12) and (13) respectively, and compared to the experimental values. These results are shown in Table 5.For contact time, the closest correspondence between the experimental data and the model predictions was obtained at the maximum velocity of $25 \mathrm{~m} / \mathrm{s}$, and the predictive ability of the equivalent linear model deteriorated somewhat as the impact velocity was decreased.

Table 5

Comparison of contact times and coefficients of restitution measured experimentally and predicted by the equivalent linear model.

\begin{tabular}{|c|c|c|c|c|}
\hline \multirow{2}{*}{$\begin{array}{l}\text { Impact } \\
\text { speed }(\mathrm{m} / \mathrm{s})\end{array}$} & \multicolumn{2}{|c|}{ Contact time (ms) } & \multicolumn{2}{|c|}{ Coefficient of restitution } \\
\hline & Experiment & Linear model & Experiment & Linear model \\
\hline \multicolumn{5}{|l|}{ Ball A } \\
\hline 5 & 2.09 & 2.42 & 0.606 & 0.649 \\
\hline 15 & 1.91 & 2.03 & 0.527 & 0.607 \\
\hline 25 & 1.91 & 1.81 & 0.462 & 0.554 \\
\hline \multicolumn{5}{|l|}{ Ball B } \\
\hline 5 & 2.09 & 2.61 & 0.640 & 0.670 \\
\hline 15 & 2.06 & 2.12 & 0.535 & 0.598 \\
\hline 25 & 1.97 & 1.91 & 0.473 & 0.554 \\
\hline \multicolumn{5}{|l|}{ Ball C } \\
\hline 5 & 1.88 & 1.97 & 0.608 & 0.641 \\
\hline 15 & 1.63 & 1.65 & 0.533 & 0.603 \\
\hline 25 & 1.50 & 1.49 & 0.488 & 0.582 \\
\hline \multicolumn{5}{|l|}{ Ball D } \\
\hline 5 & 2.09 & 2.19 & 0.636 & 0.579 \\
\hline 15 & 1.84 & 1.93 & 0.546 & 0.608 \\
\hline 25 & 1.75 & 1.73 & 0.478 & 0.558 \\
\hline
\end{tabular}

The deviations between the experimental coefficients of restitution and the equivalent model predictions showed the opposite trend, increasing with impact velocity. The model had a tendency to over-predict the coefficients of restitution,which was seen for 11 of the 12 points. The mean and largest differences between the model and experimental contact times were $7.6 \%$ and $24.4 \%$, respectively, while the mean differences when the data were partitioned by impact velocity were $12.4 \%$ at $5 \mathrm{~m} / \mathrm{s}, 3.8 \%$ at $15 \mathrm{~m} / \mathrm{s}$ and only $2.5 \%$ at $25 \mathrm{~m} / \mathrm{s}$. All of these percentages are calculated with respect to the experimental data. The corresponding mean and maximum differences for the coefficients of restitution were $13.1 \%$ and $20.1 \%$, and mean differences subdivided by particle velocity were $6.6 \%$ at $5 \mathrm{~m} / \mathrm{s}, 12.8 \%$ at $15 \mathrm{~m} / \mathrm{s}$ and $18.3 \%$ at $25 \mathrm{~m} / \mathrm{s}$. The primary explanation for the differences between the model and experimental coefficients of restitution is the use of only one value of $k$ for each ball core (Table 3 ) rather than separate values for each core type and impact velocity. Therefore the values obtained using this approach are a compromise for the three velocities assessed, as is the equivalent 
linear stiffness (Eq. (15)) and hence the coefficient of restitution (Eq. (13)).Overall, the equivalent linear model predicted the parameters of contact time and coefficient of restitution quite well.

The model confirmed that core $\mathrm{C}$ gave the shortest contact time. Furthermore, the model predicted that the coefficients of restitution decreased with increasing impact speed for all ball cores, typically from 0.64 at $5 \mathrm{~m} / \mathrm{s}$ to 0.56 at $25 \mathrm{~m} / \mathrm{s}$. Core type did not appear to be a major

factor here. As with the contact time, the coefficient of restitution did not display a large sensitivity to impact speed. From a practical viewpoint, it is useful to be able to predict the contact durations accurately using such a model as it can be problematic to measure short contact durations experimentally outside of a controlled laboratory setting.

\subsection{Parameter study}

With the developed and validated model of sliotar impact, studies were conducted to determine the sensitivity of the impact measurements (force, displacement, and contact time) to the stiffness of the ball core. For this study, the sensitivity of the maximum impact force, maximum impact displacement and the contact time during impact to the core stiffness parameter, $\mathrm{k}$, was examined. The core mass was taken to be fixed at $90 \mathrm{~g}$ and one sliotar core impact speed was selected of $15 \mathrm{~m} / \mathrm{s}$. The mean coefficient of restitution at this impact speed for the four cores was 0.5354 , which was used in the analysis (standard deviation of 0.0080 ). $\mathrm{k}$ was adjusted between 2 _ $106 \mathrm{~N} / \mathrm{m} 1.5$ and $5 \_106 \mathrm{~N} / \mathrm{m} 1.5$ as this range was realistic and the higher values might arise if cores were manufactured from a stiffer material. Fig. 6(a) illustrates how force varies with displacement and Fig. 6(b) shows how deflection and contact time vary for this range of stiffness parameters. As $\mathrm{k}$ increased, the maximum force increased, while the maximum deflection and contact time both decreased. The increasing nonlinear nature of the contact at greater values of the stiffness parameter was clearly evident. More generally, the development of a physically-based model of ball impact can be used to indicate how the parameters of stiffness and damping can be selected to improve ball performance.
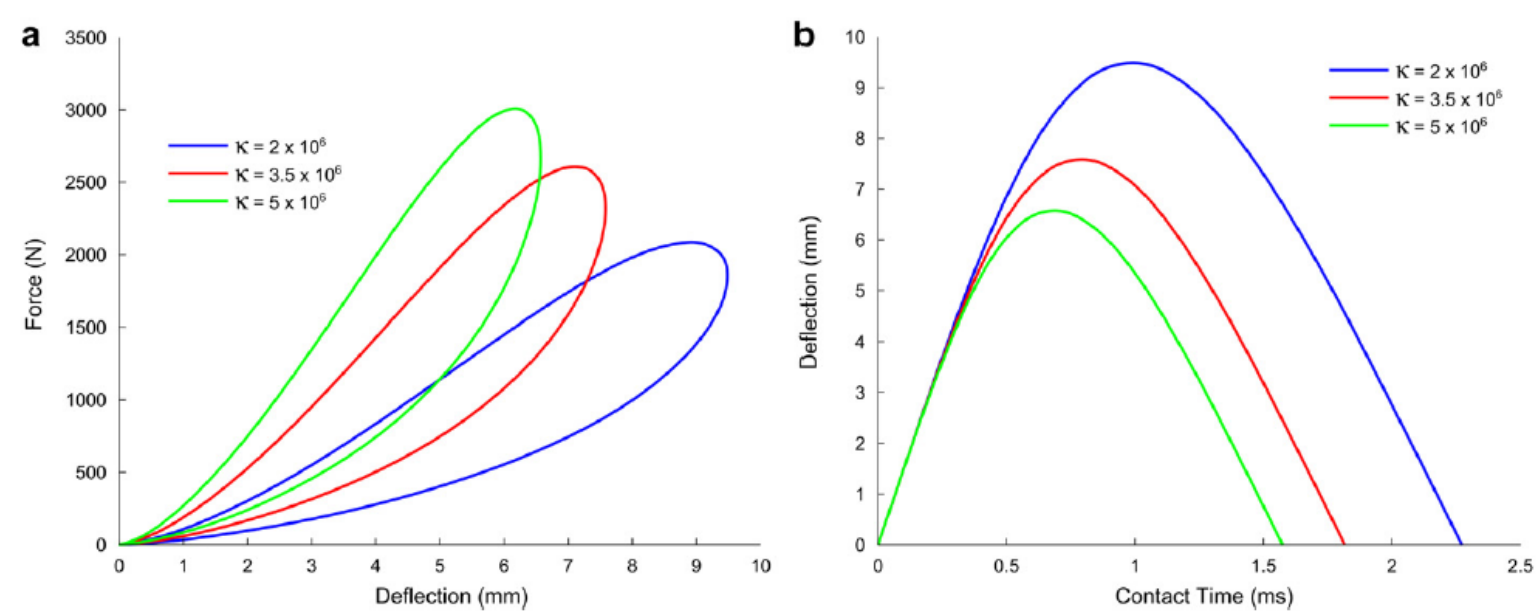

Fig. 6. Plots which illustrate the effect of changing $\kappa$ from $2 \times 10^{6} \mathrm{~N} / \mathrm{m}^{1.5}$ to $5 \times 10^{6} \mathrm{~N} / \mathrm{m}^{1.5}$ on the force, deflection and contact time for an impact at $15 \mathrm{~m} / \mathrm{s}$ : (a) force (N) versus deflection $(\mathrm{mm})$ and $(\mathrm{b})$ deflection $(\mathrm{mm})$ versus contact time $(\mathrm{ms})$. 
For example, a desirable aim is to have the coefficient of restitution as invariant as possible to promote repeatable behaviour. The coefficient of restitution falls with increasing impact velocity. The development of an expression for the coefficient of restitution in terms of equivalent linear damping and equivalent viscous damping coefficient can permit the settings of damping and stiffness that give the best control over the restitution coefficient to be estimated.

\section{Conclusions}

The objective of this study was to develop a model for sliotar core impact constitutive behaviour that required the minimum number of model parameters whilst achieving as good a fit as possible compared to the experimental data. Previous attempts at modelling ball impact were heavily reliant upon their phenomenological derivation, i.e., the modelled fit was achieved by incorporating parameters of questionable relevance. Therefore, it was regarded as highly desirable that the parameters of the model developed in this paper would be interpretable in terms of physical characteristics of the ball core. By assuming the relationship is known between manufacturing input conditions and resultant product material properties, a non-phenomenologically derived model bridges the knowledge gap between ball material physical properties and associated impact response for a range of speeds. The formation of such a parametrically-intuitive ball model would also be applicable to ball/hurley or ball/ground impacts. In this study, impact models to capture the dynamics of a collision between a sliotar core and a fixed rigid steel plate were developed and their predictions compared to experimental data. While the models required relatively few fitted constants, their output compared very well to the experimental results. The quasistatic response was described well by Hertzian theory. When the HunteCrossley models were used to predict the maximum contact force, the maximum deflection and the contact duration for each ball type and impact speed, the model outputs compared well to the measured experimental data. This can be seen in that the mean difference between experimental and model contact times was only $8.3 \%$. The samewas true for the equivalent KelvineVoigt linear model, for which the mean differences between the experimental and model contact durations and coefficients of restitution were $7.6 \%$ and $13.1 \%$, respectively.

The linear KelvineVoigt model parameters were more physically interpretable and while this model type would not generally give a good comparison with experimental results, the parameters from it permitted measured impact quantities to be well related to their effects on results. From this model, the impact velocity was found to be an important parameter in determining constitutive response. As ball impact speed increased, ball deflection increased whereas differences in contact time as a percentage of overall contact time reduced. The sensitivity of coefficient of restitution and contact time predictions did not showa significant dependence on the initial contact speed of the ball.

\section{Acknowledgements}

The authors would like to thank the Gaelic Athletic Association for their financial support for this research. 


\section{References}

[1] Gaelic Athletic Association. Official guide [part 2]. Playing rules of football and hurling. Dublin: Gaelic Athletic Association; 2008.

[2] Gaelic Athletic Association. Annual report [part 1]. http://www.gaa.ie/content/ documents/publications/annual_reports/Annual_Report_2004_Part1_Pg1_50_

100111084202.pdf; 2004 [accessed May 2011].

[3] Collins F,BrabazonD,Moran K. Viscoelastic impact characterisationof solid sports balls used in the Irish sport of hurling. Sports Engineering 2011;14(1):15e25.

[4] Carré MJ, James DM, Haake SJ. Impact of a non-homogeneous sphere on a rigid surface. Proceedings of the Institution of Mechanical Engineers, Part C: Journal of Mechanical Engineering Science 2004;218(3):273e81.

[5] Cheng N, Subic A, Takla M. Development of a fast-solving numerical model for the structural analysis of cricket balls. Sports Technology 2008;1(2):

$132 \mathrm{e} 44$.

[6] Cross R. The bounce of a ball. American Journal of Physics 1999;67(3):222e7. [7] Fuss FK. Cricket balls: construction, non-linear visco-elastic properties, quality control and implications for the game. Sports Technology 2008;1(1):41e5.

[8] Gilardi G, Sharf I. Literature survey of contact dynamics modelling. Mechanism and Machine Theory 2002;37:1213e39.

[9] Cochran AJ. Development and use of one-dimensional models of a golf ball. Journal of Sports Sciences 2002;20(8):635e41.

[10] Goodwill SR, Haake SJ. Modelling of tennis ball impacts on a rigid surface. Proceedings of the Institution of Mechanical Engineers e Part C: Journal of Mechanical Engineering Science 2004;218(10):1139e53.

[11] Collins FC. Parametric impact characterisation of a solid sports ball, with a view to developing a standard core for the GAA sliotar. PhD thesis. Dublin City University; 2011.

[12] Tanaka K, Sato F, Oodaira H, Teranishi Y, Sato F, Ujihashi S. Construction of the finiteelement models of golf balls and simulations of their collisions. Proceedings of the Institution of Mechanical Engineers, Part L: Journal of Materials: Design and Applications 2006;220(1):13e22.

[13] Cochran AJ. Club face flexibility and coefficient of restitution. In: Farrally MR, Cochran AJ, editors. Proceedings of the World Science Congress of Golf; 1998. p. 486e92.

[14] Hunt KH, Crossley FRE. Coefficient of restitution interpreted as damping in vibroimpact. ASME Journal of Applied Mechanics 1975;7:440e5.

[15] Marhefka DW, Orin DE. Simulation of contact using a nonlinear damping model. In: Proceedings of the 1996 IEEE international conference on robotics and automation; 1996. p. 1662e8. Minneapolis, Minnesota.

[16] Young WC. Roark's formulas for stress \& strain. 7th ed. New York, USA: McGrawHill; 2001. p. 25e50.

[17] Haake SJ, Carré MJ, Goodwill SR. The dynamic impact characteristics of tennis balls with tennis rackets. Journal of Sports Sciences 2003;21(10):839e50.

[18] Goodwill SR, Haake SJ. Spring damper model of an impact between a tennis ball and racket. Proceedings of the Institution of Mechanical Engineers, Part C: Journal of Mechanical Engineering Science 2001;215(11): 1331e41. 
[19] Collins F, Brabazon D, Moran K. Dynamic characterisation of high velocity ball impacts with NI LabVIEW, MAQ software and PCIe hardware. In: Baker D, editor. Proceedings of NIDays professional development conference for engineers and scientists. London: IET; November 2009. p. 14e5.

[20] Collins F, Brabazon D, Moran K. The dynamic viscoelastic characterisation of the impact behaviour of the GAA sliotar. Procedia Engineering 2010;2(2): $2991 \mathrm{e} 7$. 